\title{
On geodesic bifurcations of product spaces
}

\author{
Lenka RÝparová, Josef Mikeš and Almaz Sabykanov \\ (Presented by F. Abdullayev)
}

\begin{abstract}
The bifurcation is described as a situation where there exist at least two different geodesics going through the given point in the given direction. In the previous works the examples of a local bifurcation and closed bifurcation are constructed. This paper is devoted to further study of these bifurcations. We construct an example of an $n$-dimensional (pseudo-) Riemannian and Kählerian spaces which are product that admit local bifurcation of geodesics and that also admit closed geodesics.
\end{abstract}

2010 MSC. 53A05, 53B21, 53B30, 53B35, 53C22.

Key words and phrases. (pseudo-) Riemannian space, product space, geodesic, geodesic bifurcation.

\section{Introduction}

It is well know that on a surface there exists one and the only one geodesic going through given point in given direction. The proof of this statement follows from the analysis of system of a ordinary differential equations (ODE), and the statement is valid for surfaces for which the Christoffel symbols are differentiable functions. Kuiper [2] and Borisov [1] presented isometric imbending of class $C^{1}$.

On the other hand, if the Christoffel symbols are continuous and are not differentiable functions then the solution of above mentioned system of ODE is not unique. Therefore, there exist more than one solution of ODE and more geodesics going through given point which, at this point, have the same tangent vector. This situation is called a geodesic bifurcation.

In [4] we obtained a surface of revolution where geodesic bifurcation exists. The construction of closed geodesic bifurcation on a surface is

Research supported by IGA PrF 2018012 at Palacky University Olomouc, and by specific university research at Brno University of Technology FAST-S-18-5184. 
published in [5]. Here, we include the previously obtained results [4,5] in a short form. Furthermore, we state new results about geodesic bifurcation on product spaces. Latest results prove that there exist Kähler and also hyperbolical Kähler spaces which admit these geodesic bifurcations.

Let us note, that geodesic bifurcations were also studied in paper [6], but in this case the term geodesic bifurcation does not have the same meaning as we use in the presented work.

\section{Geodesic bifurcation}

Let $M$ be a manifold with an affine connection $\nabla$. In local chart the components $\Gamma_{i j}^{h}$ of the connection $\nabla$ are given. A curve $\gamma$ on the manifold $M$ is called geodesic if its tangent vector is recurrent along it.

Locally, on the geodesic $\gamma$ there exists a canonical parameter (arc length) $s$ for which the geodesic satisfy the equation: $\nabla_{s} \dot{\gamma}=0$. In this case, vector field $\dot{\gamma}$ is parallel along curve $\gamma$. Here "." denotes derivative with respect to arc length.

In chart $(U, x)$ above mentioned equation has the following form

$$
\ddot{x}^{h}(s)+\Gamma_{i j}^{h}(x(s)) \dot{x}^{i}(s) \dot{x}^{j}(s)=0,
$$

where $\gamma: x^{h}=x^{h}(s)$ are equation of geodesic $\gamma$ on chart $(U, x)$, and $\Gamma_{i j}^{h}$ are components of $\nabla$. Also, geodesics are often defined as curves that satisfy the equations (2.1), see [3].

We can rewrite the equations (2.1) in the form of a system of ordinary differential equations of the first order with respect to unknown functions $x^{h}(s)$ and $\lambda^{h}(s)$ like follows

$$
\dot{x}^{h}(s)=\lambda^{h}(s), \quad \dot{\lambda}^{h}(s)=-\Gamma_{i j}^{h}(x(s)) \dot{x}^{i}(s) \dot{x}^{j}(s),
$$

where $\lambda^{h}(s)$ is a tangent vector of the curve $\gamma(s)$ at the point $x^{h}(s)$. For the initial conditions

$$
x^{h}(s)=x_{0}^{h}, \quad \lambda^{h}(s)=\lambda_{0}^{h}
$$

the solution of the system (2.2) is a geodesic going through the point $x_{0}^{h}$ in the direction $\lambda_{0}^{h}(\neq 0)$. If the function $\Gamma_{i j}^{h}(x)$ are continuous functions, then from the general theory of differential equations follows that the equations (2.2) with initial conditions (2.3) have solution. Furthermore, if the functions $\Gamma_{i j}^{h}(x)$ belong to differentiability class $C^{1}$ then the solution of such system is unique. It is worth to note that the last condition of differentiability of the functions $\Gamma_{i j}^{h}(x)$ can be replaced with the Lipschitz condition. 
In [3] there is given an example of the space with affine connection where the components are only continuous functions. In this case, there exist one and only one geodesic passing through given point $x_{0}^{h}$ in given direction $\lambda_{0}^{h}$.

Example 2.1. Let us consider a space $A_{n}$ with affine connection $\nabla$ defined in a certain coordinate neighbourhood $(U, x)$ by its continuous components

$$
\Gamma_{h h}^{h}=f^{h}\left(x^{h}(s)\right), \quad h=1, \ldots, n,
$$

the other components are vanishing. The solution of the Cauchy problem (2.2) and (2.3) was obtained in the following explicit form

$$
\int_{x_{0}^{h}}^{x^{h}(s)} \exp \left(\int_{x_{0}^{h}}^{w} f^{h}(\tau) \mathrm{d} \tau\right) \mathrm{d} w=\lambda_{0}^{h} s .
$$

This solution is unique and exists in the whole neighbourhood $U$, that is the geodesic passing through the point $x_{0}^{h}$ in direction $\lambda_{0}^{h}$ is also unique. Let us remind that in this example the components of the connection were continuous but not differentiable functions.

It was shown [4], that there exist a surface of revolution which admits geodesic bifurcation.

Example 2.2. Let us consider a surface of revolution $\mathcal{S}$ given by the equations

$$
x=r(u) \cos v, \quad y=r(u) \sin v, \quad z=z(u),
$$

where

$$
r(u)=\frac{1}{\sqrt{1-u^{2 \alpha}}}, \quad z(u)=\int_{0}^{u} \sqrt{1-\alpha^{2} t^{4 \alpha-2} \cdot\left(1-t^{2 \alpha}\right)^{-3}} \mathrm{~d} t,
$$

$u \in(-1,1), v \in(-\pi, \pi)$ are parameters.

For this surface the Christoffel symbols are continuous functions which are evidently not differentiable and also do not satisfy Lipschitz condition. Then on the surface of revolution $\mathcal{S}$ there exist geodesic bifurcations for $\alpha \in(0,1)$.

The proof of this statement follows from the existence of two different geodesics going through the point $(0,0)$ in the direction $(0,1)$. These geodesics are given by the equations

$$
\begin{aligned}
\text { I. } & u=0, \quad v=s \\
\text { II. } & u=((1-\alpha) s)^{\frac{1}{1-\alpha}}, \quad v=s-\frac{((1-\alpha) s)^{\frac{1+\alpha}{1-\alpha}}}{1+\alpha} .
\end{aligned}
$$


The first one we shall call a trivial geodesic - also called gorge circle and the second one nontrivial geodesic passing through the point $(0,0)$ in the direction $(0,1)$.

We note a remarkable fact that the consequence of this statement is that geodesic bifurcation exists in each point of the gorge circle since the given surface of revolution is symmetric. Furthermore, there exists an infinite number of geodesics going through the point $(0,0)$ in direction $(0,1)$. Moreover, the surface $\mathcal{S}$ has the metric $\mathrm{d} s^{2}=\mathrm{d} u^{2}+r^{2}(u) \mathrm{d} v^{2}$ and because any surface with this metric admits a nontrivial geodesic mapping, then projective corresponding spaces preserve above mentioned geodesic bifurcations.

Detailed description of this problem can be found in [4].

The previously obtained results are further developed in [5]. In this paper there is constructed a surface on which bifurcation of closed geodesics exists.

Example 2.3. Let us consider a certain neighbourhood of the gorge circle of the surface $\mathcal{S}$ mentioned in the previous example, where the geodesic bifurcations exists. Let us suppose that the geodesic curve $\gamma$ starts at the point $(0,0)$ and has a tangent vector $(0,1)$, the "end" lies also on the gorge circle, and is part of another nontrivial geodesic.

To construct a surface where bifurcation of closed geodesic exists, the goal is to connect the ends of the geodesic $\gamma$ with the smooth curve which would form the surface. In this construction is used a fact that the surface of revolution is symmetric and also results obtained in [4].

For further information and more detailed description of the above mentioned construction of this example see [5].

\section{Product manifolds}

A Riemannian manifold $\mathbb{V}_{n}$ is called a product manifold of Riemannian manifolds $\stackrel{1}{\mathbb{V}}{ }_{n_{1}}, \stackrel{2}{\mathbb{V}}_{n_{2}}, \ldots, \stackrel{m}{\mathbb{V}}_{n_{m}}\left(n_{1}+n_{2}+\cdots+n_{m}=n\right)$

$$
\mathbb{V}_{n}=\stackrel{1}{\mathbb{V}}_{n_{1}} \otimes \stackrel{2}{\mathbb{V}}_{n_{2}} \otimes \cdots \otimes \stackrel{m}{\mathbb{V}}_{n_{m}}
$$

if the metrics are related by

$$
g=g_{1} \otimes g_{2} \otimes \cdots \otimes g_{m} .
$$

Locally this means that there exists a coordinate system $\left(x^{i}\right)$ such that the metric forms of these Riemannian manifolds satisfy

$$
\mathrm{d} s^{2}=\mathrm{d} s_{1}^{2}+\mathrm{d} s_{2}^{2}+\ldots+\mathrm{d} s_{m}^{2},
$$


where

$$
\begin{gathered}
\mathrm{d} s^{2}=g_{i j}\left(x^{k}\right) \mathrm{d} x^{i} \mathrm{~d} x^{j} \quad \text { and } \quad \mathrm{d} s_{\sigma}^{2}={\stackrel{\sigma}{g_{i_{\sigma} j_{\sigma}}}}\left(x^{k_{\sigma}}\right) \mathrm{d} x^{i_{\sigma}} \mathrm{d} x^{j_{\sigma}}, \\
i, j, k=\langle 1, n\rangle ; \quad i_{\sigma}, j_{\sigma}, k_{\sigma}=\left\langle p_{\sigma}, r_{\sigma}\right\rangle ; \\
1=p_{1} \leq r_{1}<p_{2} \leq r_{2}<\cdots<p_{m} \leq r_{m}=n .
\end{gathered}
$$

It is know [3, pp. 192] that a product manifold $\mathbb{V}_{n}$ with metric

$$
\bar{g}=\left(\alpha_{1} g_{1}\right) \otimes\left(\alpha_{2} g_{2}\right) \otimes \cdots \otimes\left(\alpha_{m} g_{m}\right), \quad \text { where constants } \quad \alpha_{\sigma} \neq 0
$$

admit affine mappings $f: \mathbb{V}_{n}(M, g) \longrightarrow \overline{\mathbb{V}}_{n}(M, \bar{g})$.

By analysing equations (2.1) we can verify that the geodesic $\gamma$ in product manifold $\mathbb{V}_{n}=(M, g)$ can be generated by geodesic $\stackrel{i}{\gamma} \subset \stackrel{i}{V}_{n}$ for $i=1,2, \ldots m$ like follows

$$
\gamma=\stackrel{1}{\gamma} \otimes \stackrel{2}{\gamma} \otimes \cdots \otimes \stackrel{m}{\gamma}
$$

while among these geodesics $\stackrel{i}{\gamma}$ there can exist "trivial geodesics" which are points in the space, i.e. they are defined by the equations $x^{k_{i}}=\underset{0}{x^{k_{i}}}=$ const.

Kähler manifold $K_{n}$ is a Riemannian space with a metric $g$ and a structure $F$ that satisfies following conditions

$$
F^{2}=-\mathrm{Id}, \quad g(X, F X)=0, \quad \nabla F=0,
$$

for any vector $X$, where $\nabla$ is Levi-Civita connection of the space.

It can be easily verified that the product space of the Kählerian spaces

$$
K_{n}=\stackrel{1}{K} \otimes \stackrel{2}{K} \otimes \cdots \otimes \stackrel{m}{K}
$$

is also Kählerian space with structure which have analogical construction

$$
F=\stackrel{1}{F} \otimes \stackrel{2}{F} \otimes \cdots \otimes \stackrel{m}{F}, \quad \text { i.e. } \quad F_{i_{\sigma}}^{h_{\sigma}}=\stackrel{\sigma_{F}}{i_{\sigma}},
$$

for $\sigma=1, \ldots, m$, and the other components of the structure $F$ are vanishing.

From the other side, two dimensional Riemannian manifold is always Kähler, see [3, pp. 130], where the following holds

$$
F_{i}^{h}=\varepsilon_{i j} g^{j h}, \quad \varepsilon_{i j}=\sqrt{g_{11} g_{22}-g_{12}^{2}} \cdot\left(\begin{array}{cc}
0 & 1 \\
-1 & 0
\end{array}\right) .
$$




\section{Geodesic bifurcation of product spaces}

The above mentioned construction of the product spaces can be now used for a similar construction of geodesic bifurcation in $n$-dimensional Riemannian spaces and also in Kähler spaces.

Evidently, it is sufficient to take take one from the components $\stackrel{i}{\mathbb{V}}$ of the above described product space (3.1) as the space with metric described in examples 2.2 and 2.3. For example, if $\stackrel{1}{\mathbb{V}}$ is the space with the metric described in examples 2.2 and 2.3 and this space has a geodesic bifurcation at the point $\underset{\gamma}{\gamma}(0)$ then it is obvious that geodesic

$$
\gamma=\stackrel{1}{\gamma} \otimes \stackrel{2}{\gamma} \otimes \cdots \otimes \stackrel{m}{\gamma}
$$

has a geodesic bifurcation at the point $\gamma(0)$.

From the above mentioned follows that in this manner it is possible to construct an $n$-dimensional Riemannian spaces where the geodesic bifurcations exist. Analogically, we can contruct an $n$-dimensional Kählerian spaces which also admit geodesic bifurcations.

Let us note that the geodesic bifurcations do not have to exist in other spaces $\stackrel{2}{\mathbb{V}}, \ldots, \stackrel{m}{\mathbb{V}}$. In case there exists a geodesic bifurcation at the point $\stackrel{i}{\gamma}(0)$ of the space $\stackrel{i}{\mathbb{V}}$ then the image of the geodesic in the product space $\mathbb{V}_{n}$ would be much more complicated.

Using global results about geodesic bifurcations obtained and described in previous paper [5] where the bifurcations of closed geodesic are constructed, it is also possible to construct bifurcation of closed geodesics in n-dimensional Riemannian and Kähler manifolds.

In addition, the construction of the product spaces makes it possible to construct also pseudo-Riemannian spaces where there exist geodesic bifurcations of isotropic geodesics, i.e. geodesic which have vanishing length.

Example 4.1. Let $V=\stackrel{1}{\mathbb{V}} \otimes \stackrel{2}{\mathbb{V}}$ be product space of the spaces $\stackrel{1}{\mathbb{V}}$ and $\stackrel{2}{V}$ and let $g_{i_{1} j_{1}}, i_{1}, j_{1}=1,2 ; g_{i_{2} j_{2}}, i_{2}, j_{2}=1,2$ be positive and negative metric forms of spaces $\mathbb{V}, \mathbb{V}$ respectively.

In Riemannian space $\stackrel{1}{\mathbb{V}}$ the geodesic $\stackrel{1}{\gamma}$ has a bifurcation at point ${ }_{\gamma}^{1}(0)$ (space $\mathbb{V}$ can be the space from the examples 2.2 and 2.3 ). Then 
the length of the vector is $\left|\frac{1}{\gamma}(0)\right|=1$. Let us suppose that in Riemannian space $\stackrel{2}{\mathbb{V}}$ there exists a geodesic $\stackrel{2}{\gamma}$ for which $\left|{ }_{\gamma}^{2}(0)\right|=1$. Then the geodesic $\gamma$ for which

$$
x^{i_{1}}=x_{1}^{i_{1}} \quad \text { and } \quad x^{i_{2}}=\underset{2}{x^{i_{2}}}
$$

has tangent vectors $\lambda^{i}$ for which

$$
\lambda^{i_{1}}=\mathrm{d}_{1} x_{1}^{i_{1}} / \mathrm{d} t \quad \text { and } \quad \lambda^{i_{2}}=\mathrm{d} x_{2}^{i_{2}} / \mathrm{d} t .
$$

Since $g_{i_{1} j_{1}}$ is a positive form and $g_{i_{2} j_{2}}$ is a negative form, it is evident that $|\lambda|=0$ because

$$
|\lambda|=\sqrt{g_{i j} \lambda^{i} \lambda^{j}}=\sqrt{g_{i_{1} j_{1}} \lambda^{i_{1}} \lambda^{j_{1}}+g_{i_{2} j_{2}} \lambda^{i_{2}} \lambda^{j_{2}}}=\sqrt{1-1}=0 .
$$

\section{Conclusion}

The work develops the results and ideas from the previously published papers $[4,5]$. In presented work we have constructed an $n$-dimensional Riemannian product spaces and also Kählerian product spaces which admit local geodesic bifurcations and also bifurcations of closed geodesic.

\section{References}

[1] Y. F. Borisov, Irregular $C^{1, \beta}$-surfaces with an analytic metric // Siberian Math. J., 45 (2004), N. 1, 19-52.

[2] N. H. Kuiper, On $C^{1}$-isometric imbeddings. I // Proc. Koninkl. nederl. akad. wetensch. A, 58 (1955), 545-556.

[3] J. Mikeš et al., Differential geometry of special mappings, Palacky Univ. Press, Olomouc, 2015.

[4] L. Rýparová, J. Mikeš, On geodesic bifurcations // Geometry, integrability and quantization, 18 (2017), 217-224.

[5] L. Rýparová, J. Mikeš, Bifurcation of closed geodesics // Geometry, integrability and quantization, 19 (2018), 188-192.

[6] H. Thielhelm, A. Vais, F. E. Wolter, Geodesic bifurcation on smooth surfaces // The Visual Computer, 31 (2015), N. 2, 187-204. 
Contact information

Lenka Rýparová Department of Algebra and Geometry, Palacky University Olomouc, Olomouc, Czech Republic E-Mail: lenka.ryparova01@upol.cz

Josef Mikeš

Department of Algebra and Geometry, Palacky University Olomouc, Olomouc, Czech Republic E-Mail: josef.mikes@upol.cz

\section{Almaz Sabykanov}

Department of Geometry, Kyrgyz National University, Bishkek, Kyrgyzstan

E-Mail: almazbek.asanovich@mail.ru 